\title{
UNSTABLE RESISTANCE TO METHICILLIN \\ IN STAPHYLOCOCCUS EPIDERMIDIS
}

\author{
D. I. ANNEAR AND W. B. GRUBB \\ Department of Microbiology, Royal Perth Hospital, \\ and Department of Microbiology, The University of Western Australia
}

\section{Plate XII}

IN Staphylococcus aureus, resistance to several antibiotics is controlled by extrachromosomal elements (plasmids). Reports now suggest that, in some strains, resistance to methicillin may also be plasmid controlled. The evidence for this is the loss of resistance in cultures which have been (1) treated with acriflavine (Dornbusch, Hallander and Löfquist, 1969), (2) grown for prolonged periods at elevated temperature (Al Salihy and James, 1972), or (3) grown and stored at room temperature (Grubb and Annear, 1972; Lacey, 1972; Annear and Grubb, 1973a). The purpose of this present paper is to report a possible plasmid location for methicillin resistance in a strain of $S$. epidermidis. Schaefler (1972) reported simultaneous loss of resistance to oxacillin and kanamycin in $S$. epidermidis; and independent loss of penicillinase production (Baldwin, Strickland and Cox, 1969; Schaefler) and of tetracycline and chloramphenicol resistance (Bentley, Hahn and Lepper, 1970; Schaefler) has also been described.

The strain investigated here (no. S34) had been studied previously in relation to the " optimal zone" of sensitivity that it shows towards methicillin (Annear and Grubb, 1973b); although resistant to high concentrations of methicillin, it was inhibited over a narrow range of intermediate concentrations of the antibiotic, and in consequence showed a characteristic annular area of inhibition in disk-diffusion tests (Annear, 1972). During the early stages of this work it became apparent that after the organism had been subcultured at irregular intervals for several weeks, the fraction of methicillin-resistant cells in the population became significantly reduced. Further investigation indicated that sensitive variants were being spontaneously produced during growth or maintenance on culture media.

This instability was examined more formally by serial subculture of resistant colonies. The antibiotic-sensitivity patterns of the methicillinresistant and -sensitive organisms were studied with special reference to methicillin and benzylpenicillin. The organisms were also compared with respect to the "optimal zone" phenomenon with methicillin and the antagonism of this by other penicillins and cephalosporins (Annear and Grubb, 1973b). Dried cultures (desiccates) were prepared at critical points of the experiments to safeguard against loss or contamination, to serve as reference material for 
further investigation, and to obviate the necessity for immediate screening (Annear and Grubb, 1973c).

\section{MATERIALS AND METHODS}

Organisms. The test organism was $S$. epidermidis, strain S34. The methicillin-resistant form is designated S34/R and the sensitive variant S34/S.

Media and antibiotics. The two agar media used were: (1) medium A, 1 per cent. Bacto Tryptose (Difco), 1.5 per cent. Bacto Agar (Difco), $p \mathrm{H} \mathrm{6.8;} \mathrm{and} \mathrm{(2)} \mathrm{medium} \mathrm{B,} \mathrm{Blood} \mathrm{Agar}$ Base no. 2 (Oxoid). Medium $A$ was used because of its suitability for the demonstration of the optimal zone of methicillin activity on the resistant form of the organism. It was also the medium on which instability of methicillin resistance was first observed and was used thereafter for studying this. The optimal zone of inhibition of methicillin-resistant organisms was also shown in previous work to vary in clarity with different batches of the drug. A batch that showed it clearly (batch A, Annear and Grubb, 1973b) was used in the present study.

Unstable resistance. The general procedure for demonstrating the instability of methicillin resistance was as described previously for other antibiotics (Annear and Grubb, 1972). In outline, it involved demonstrating that resistant colonies, when serially subcultured, consistently yield sensitive variants in each cycle. The multipoint screening method used in this work was convenient, but carried some risk of cross contamination of the "spots", so compromising studies on the progeny. Although the risk of false results may be reduced to negligible proportions by repetitive serial screening, we now consider it sounder policy to work with single colonies derived from the spots, rather than with the spots themselves. The colonies are checked for resistance before continuing with the procedure. In the present study therefore, representative resistant and sensitive colonies were isolated. The resistant colonies were grown and kept on slopes of medium $A$ at room temperature for each cycle of testing. At varying intervals, the slopes were subcultured onto an agar plate, then single colonies were prepared from this fresh growth and were screened for the presence of sensitive variants. Screening for methicillin resistance was done in medium A at a drug concentration of $25 \mu \mathrm{g}$ per $\mathrm{ml}$.

Antagonism of methicillin activity. Methods for demonstrating these effects have been described elsewhere (Annear and Grubb, 1973b).

Testing for sensitivity to methicillin and penicillin. The minimum inhibitory concentration (MIC) of these antibiotics was determined by surface inoculation of cultures onto antibioticagar plates. Two levels of inoculum were used; the "heavy" inoculum was one drop of neat broth culture and the " light " inoculum a drop containing approximately 100 organisms.

Preparation of desiccates. Suspensions were made by harvesting growth from medium $B$ in 5 per cent. sodium glutamate, and desiccates were prepared on ceramic beads and stored at $4^{\circ} \mathrm{C}$ (Annear and Grubb, 1973c).

For each cycle of the experiments, desiccates representing the following cultures were prepared: (1) the initial resistant organism; (2) the subculture derived from it containing a mixture of resistant organisms and sensitive variants; (3) resistant organisms obtained from (2) to be used for the subsequent cycle; (4) sensitive variants obtained from (2).

\section{RESULTS \\ Instability of methicillin resistance}

Serial subculture of resistant colonies for three successive cycles consistently revealed unstable methicillin resistance. Slopes were screened at intervals during each cycle until sensitive variants formed at least 20 per cent. of the population. Table I shows the wide variation in the proportion of sensitive variants present in agar-slope cultures prepared from three different colonies 


\section{S34/R}

S34/S
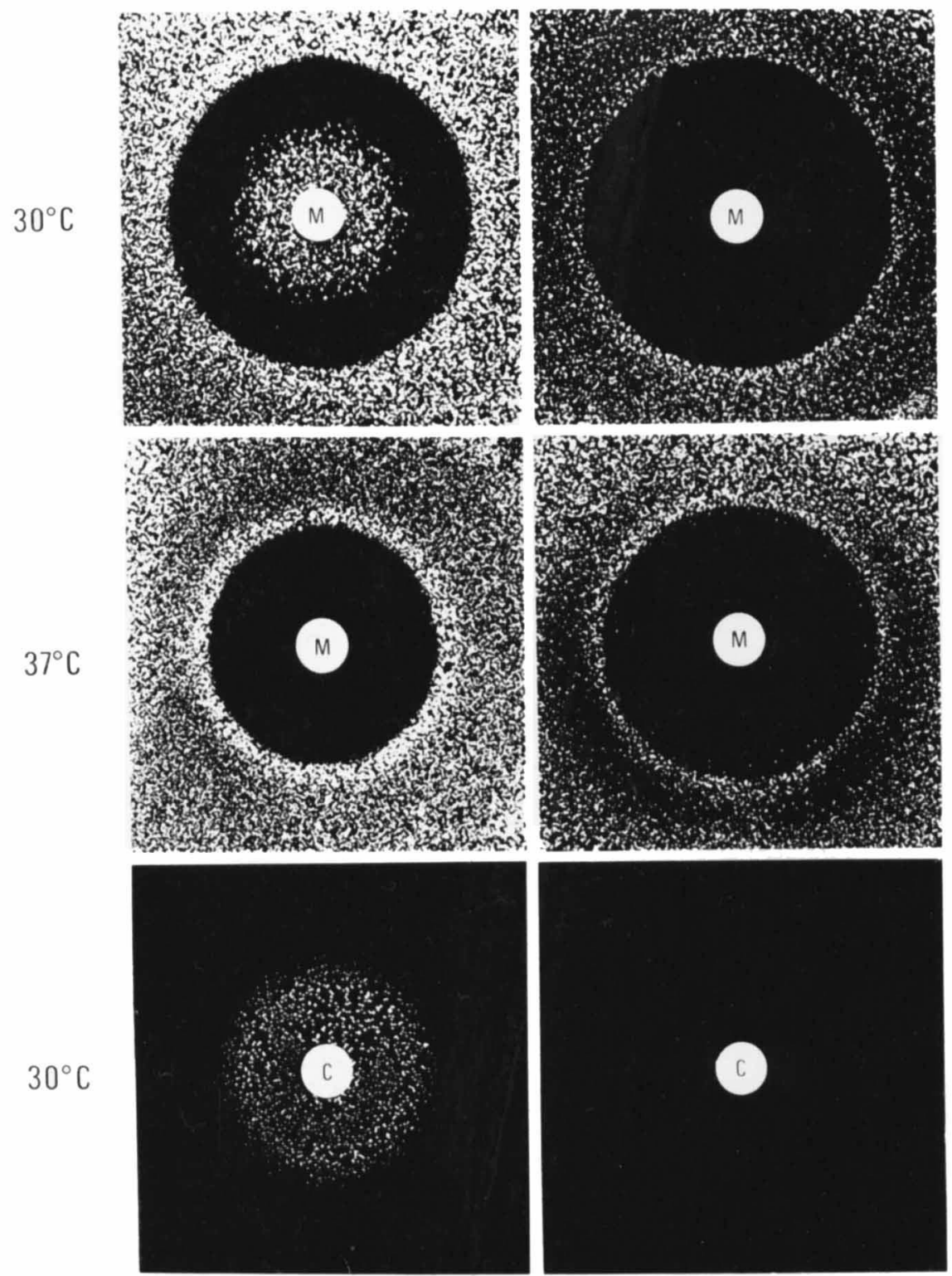

FIGURE.-Disk-diffusion tests on Staphylococcus epidermidis strain S34/R and its methicillin-sensitive variant S34/S. The top and centre pictures illustrate tests with methicillin disks (M: $20 \mu \mathrm{g}$ per disk) on nutrient agar (medium A). The "optimal zone" of inhibition of the methicillinresistant organism is apparent only at $30^{\circ} \mathrm{C}$. The bottom pictures show the effect of cephaloridine disks (C: $0.01 \mu \mathrm{g}$ per disk) on the inhibition of the organisms by methicillin $(2 \mu \mathrm{g}$ per $\mathrm{ml})$ in medium A; the inhibitory effect is antagonised for the methicillin-resistant organism but not for the methicillin-sensitive variant. 
and stored at room temperature in parallel. Once the variants began to appear there was a steady increase in their frequency with time.

TABLE I

Proportion of methicillin-sensitive variants in agar-slope cultures prepared from three resistant colonies of Staphylococcus epidermidis (strain S34) and stored at room temperature

\begin{tabular}{c|rrr}
\hline Days of storage & \multicolumn{4}{|c}{$\begin{array}{c}\text { Sensitive variants per } \\
\text { in culture prepared from colonies }\end{array}$} \\
\hline $\begin{array}{r}\text { A } \\
20\end{array}$ & B & C \\
20 & 0 & 0 & 0 \\
30 & 0 & 0 & 0 \\
40 & 3 & 0 & 12 \\
50 & 20 & 6 & 46 \\
\hline
\end{tabular}

Resistance of organisms $S 34 / R$ and $S 34 / S$ to methicillin

Methicillin resistance was measured on the two media at $30^{\circ} \mathrm{C}$ and $37^{\circ} \mathrm{C}$ with light and heavy inocula. The results shown in table II are those for medium B. With medium A the results were similar, except that the light inoculum of organism S34/R gave an MIC value of $>1000 \mu \mathrm{g}$ per ml at $30^{\circ} \mathrm{C}$.

TABLE II

Minimum inhibitory concentrations (MIC) of methicillin for $S$. epidermidis, strain $S 34 / R$, and its sensitive variant $S 34 / S$ on medium $B$

\begin{tabular}{l|cccc}
\hline Organism & $\overbrace{\begin{array}{c}\text { with } \\
\text { light inoculum }\end{array}}^{\text {at } \begin{array}{c}\text { with } \\
\text { heavy inoculum }\end{array}}$ & $\begin{array}{c}\text { with } \\
\text { light inoculum }\end{array}$ & $\begin{array}{c}\text { at } \begin{array}{c}\text { with } \\
\text { weavy inoculum } \\
\text { S34/R }\end{array} \\
\text { S34/S }\end{array}$ \\
\hline 250 & $>1000$ & 4 & $>1000$ \\
\hline
\end{tabular}

The variant S34/S was clearly sensitive under all conditions tested, whereas organism S34/R showed high methicillin resistance except when a light inoculum was tested at $37^{\circ} \mathrm{C}$. Table III shows the optimal zone of methicillin activity obtained with a light inoculum of the resistant organism S34/R on medium A at $30^{\circ} \mathrm{C}$ and the resistance of the organism to higher concentrations of the same drug. This effect was not seen in tests with a heavy inoculum. The sensitive variant showed an unequivocal sensitivity to methicillin under the same conditions. The results of the agar-diffusion test (the figure) are in close agree- 
ment with those obtained in the corresponding agar-dilution tests and show clearly the optimal zone of methicillin activity obtained with the resistant organism S34/R at $30^{\circ} \mathrm{C}$. At $37^{\circ} \mathrm{C}$, clear zones of sensitivity were obtained with both organisms S34/R and S34/S.

TABLE III

Effect of methicillin* on S. epidermidis (strain S34/R) and its sensitive variant (S34/S)

\begin{tabular}{|c|c|c|c|c|c|c|c|c|c|c|c|}
\hline \multirow{2}{*}{ Organism } & \multicolumn{11}{|c|}{ Amount of growth in the presence of methicillin ( $\mu \mathrm{g}$ per $\mathrm{ml})$} \\
\hline & 1000 & 250 & 64 & 16 & 8 & 4 & 2 & 1 & 0.5 & $0 \cdot 25$ & 0.00 \\
\hline S34/R & ++ & ++ & ++ & ++ & + & - & - & + & ++ & ++ & $+t$ \\
\hline $\mathrm{S} 34 / \mathrm{S}$ & - & - & - & - & - & - & - & + & ++ & $t+$ & ++ \\
\hline
\end{tabular}

* Titrations on medium $\mathrm{A}$ at $30^{\circ} \mathrm{C}$; light inoculum. growth.

$++=$ No inhibition of growth; $+=$ some inhibition of growth; $--=$ complete inhibition of

\section{Penicillin resistance}

The effects of benzylpenicillin in agar-dilution tests in medium B on organisms S34/R and S34/S are shown in table IV. Similar results were obtained on medium $\mathrm{A}$, and each medium gave similar results at $30^{\circ} \mathrm{C}$ and $37^{\circ} \mathrm{C}$. The results show a clear difference between organisms S34/R and S34/S when

\section{TABLE IV}

Minimum inhibitory concentration (MIC) of benzylpenicillin for $S$. epidermidis $S 34 / R$ and its sensitive variant $S 34 / S$ on medium $B$ at $37^{\circ} \mathrm{C}$

\begin{tabular}{lcc}
\hline Organism & \multicolumn{2}{c}{ MIC of penicillin $(\mu \mathrm{g}$ per $\mathrm{ml})$ for } \\
& 64 & 250 \\
$\mathrm{~S} 34 / \mathrm{R}$ & 0.25 & 500 \\
$\mathrm{~S} 34 / \mathrm{S}$ & & heavy inoculum \\
\hline
\end{tabular}

light inocula were used; with heavy inocula, however, both showed high resistance, S34/S showing a small but consistently higher resistance than S34/R. On medium A this difference was four-fold.

Results with S. aureus (NCTC no. 6571)

Both light and heavy inocula of this organism were used as controls in all the agar dilution studies of antibiotic resistance. For penicillin, all MIC values were approximately $0.01 \mu \mathrm{g}$ per $\mathrm{ml}$, while for methicillin they were approximately $0.5 \mu \mathrm{g}$ per $\mathrm{ml}$. No anomalous results were observed. 


\section{Absence of optimal zone of methicillin activity in the presence of cephaloridine}

The figure also shows the antagonistic effect of cephaloridine on the inhibition of growth of the resistant strain S34/R by moderate concentrations of methicillin and the absence of any such effect with the sensitive variant S34/S. The concentration of methicillin in the test $(2 \mu \mathrm{g}$ per $\mathrm{ml})$ is in the region of the MIC for S34/S and at the lower end of the optimal zone for S34/R (table II).

\section{Sensitivity to other antibiotics}

Strains S34/R and S34/S were screened by disk-diffusion methods with a limited range of other antibiotics. Both were sensitive to kanamycin, streptomycin, gentamicin, and lincomycin, and resistant to erythromycin and to tetracycline.

\section{DisCUSSION}

In a previous study (Annear and Grubb, 1973b) and in the present one, $S$. epidermidis strain S34 has shown an optimal zone of sensitivity when tested against methicillin. Apart from this, the organism exhibits an extremely high resistance to the drug. The present work shows quite clearly that this resistance is unstable and that it is slowly but consistently lost during growth and maintenance on agar medium at room temperature. The results suggest that methicillin resistance is controlled by an extrachromosomal determinant (plasmid).

The findings here are representative of those obtained with numerous singlecolony isolates of the resistant and the sensitive organism. These differ in several respects. The response of the resistant organism (S34/R) to methicillin is modified markedly by inoculum size, medium and temperature, and in addition it exhibits the as yet unexplained anomalous zone of inhibition by intermediate concentrations of methicillin and the antagonism of this effect by other penicillins and cephalosporins. The sensitive variant (S34/S) by contrast exhibits a relatively simple response to the drug and one not markedly modified by the factors that affect the resistance of strain S34/R.

The resistance of the variant $S 34 / \mathrm{S}$ to benzylpenicillin is similar to that of the common hospital penicillinase-producing $S$. aureus, i.e., a heavy inoculum is highly resistant whereas a light one is relatively sensitive. Strain S34/R shows little inoculum effect with benzylpenicillin, and both light and heavy inocula are markedly resistant; in this respect it resembles a methicillinresistant $S$. aureus. One particularly puzzling fact is that, when heavy inocula are compared, the S34/S variant is consistently more penicillin resistant than strain S34/R. This reversal of the results obtained with light inocula is a paradox that may be resolved when more is known about the $\beta$-lactamase activity of the two organisms. Attempts so far to investigate this have yielded equivocal results.

Although numerous isolates of $S$. epidermidis have been shown to exhibit an anomalous resistance to methicillin (Annear, 1972), instability of this drug resistance has so far been demonstrated in only one strain. More work is needed to determine whether the two phenomena are consistently related. No 
work has yet been done to determine conditions under which loss of methicillin resistance in this organism may be accelerated, but recent results with $S$. aureus (Annear and Grubb, 1973a) suggest that growth in liquid media may do so.

\section{SUMMARY}

Unstable resistance to methicillin has been demonstrated in an isolate of Staphylococcus epidermidis. Loss of resistance occurs rather slowly but consistently on nutrient agar kept at bench temperature. The resistant organism exhibits an "optimal zone" of inhibition by intermediate concentrations of the drug, and this is antagonised by cephalosporins and other penicillins. These effects are markedly modified by the medium, inoculum size and temperature. Neither of the phenomena has been observed with the sensitive variant. Against penicillin there is a paradoxical effect in that with light inocula the methicillin-resistant organism is much more resistant to penicillin than its sensitive variant, whereas the reverse holds for heavy inocula.

\section{REFERENCES}

al SAlihy, Sabria, M., AND James, A. M. 1972. Loss of methicillin-resistance from resistant strains of Staphylococcus aureus. Lancet, $2,331$.

ANNEAR, D. I. 1972. An optimal zone of methicillin activity with Staphylococcus epidermidis. Med. J. Aust., 1, 413.

ANNEAR, D. I., AND GRUBB, W. B. 1972. Linked and unstable resistance to kanamycin and penicillin, and diffusible pigment production, in an isolate of Staphylococcus aureus. J. Med. Microbiol., 5, 109.

ANNEAR, D. I., AND GrubB, W. B. 1973a. Spontaneous loss of methicillin resistance in Staphylococcus aureus. Lancet, 1, 110.

ANNEAR, D. I., AND GRUBb, W. B. 1973b. Optimal zone of methicillin activity for Staphylococcus epidermidis and its antagonism by other penicillins and cephalosporins. Med. J. Aust., 1, 160.

Annear, D. I., AND GrubB, W. B. 1973c. Preservation of Staphylococcus aureus with unstable antibiotic resistance by drying. J. Hyg., Camb., 71, 411.

Baldwin, J. N., Strickland, R. H., ANd Cox, Marilyn F. 1969. Some properties of the beta-lactamase genes in Staphylococcus epidermidis. Appl. Microbiol., 18, 628.

Bentley, D. W., HaHN, J. J., AND LePPER, M. H. 1970. Transmission of chloramphenicolresistant Staphylococcus epidermidis: epidemiologic and laboratory studies. J. Infect. Dis., 122, 365.

Dornbusch, Katharine, Hallander, H. O., and Löfquist, F. 1969. Extrachromosomal control of methicillin resistance and toxin production in Staphylococcus aureus. $J$. Bact., 98, 351 .

GrubB, W. B., and ANNeAR, D. I. 1972. Spontaneous loss of methicillin resistance in Staphylococcus aureus at room temperature. Lancet, 2, 1257.

LACEY, R. W. 1972. Genetic control in methicillin-resistant strains of Staphylococcus aureus. J. Med. Microbiol., 5, 497.

SCHAEfler, S. 1972. Polyfunctional penicillinase plasmid in Staphylococcus epidermidis: bacteriophage restriction and modification mutants. J. Bact., 112, 697. 